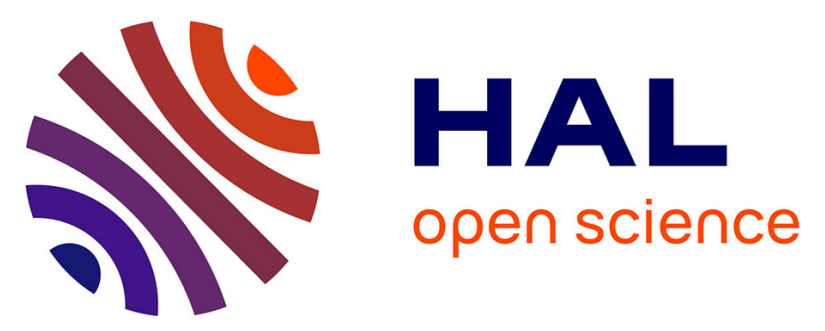

\title{
Fatigue crack initiation around inclusions for a carbon black filled natural rubber: an analysis based on micro-tomography
}

Thomas Glanowski, Yann Marco, V. Le Saux, Bertrand Huneau, Clément Champy, Pierre Charrier

\section{To cite this version:}

Thomas Glanowski, Yann Marco, V. Le Saux, Bertrand Huneau, Clément Champy, et al.. Fatigue crack initiation around inclusions for a carbon black filled natural rubber: an analysis based on microtomography. 11th European Conference on Constitutive Models for Rubber (ECCMR 2019), Jun 2019, Nantes, France. 10.1201/9780429324710 . hal-02304555

HAL Id: hal-02304555

https://hal-ensta-bretagne.archives-ouvertes.fr/hal-02304555

Submitted on 11 Oct 2019

HAL is a multi-disciplinary open access archive for the deposit and dissemination of scientific research documents, whether they are published or not. The documents may come from teaching and research institutions in France or abroad, or from public or private research centers.
L'archive ouverte pluridisciplinaire HAL, est destinée au dépôt et à la diffusion de documents scientifiques de niveau recherche, publiés ou non, émanant des établissements d'enseignement et de recherche français ou étrangers, des laboratoires publics ou privés. 


\title{
Fatigue crack initiation around inclusions for a carbon black filled natural rubber: an analysis based on micro-tomography
}

\author{
T. Glanowski \\ IRDL/Vibracoustic, CAE \& Durability Prediction, Carquefou, France
}

Y. Marco \& V. Le Saux

Institut de Recherche Dupuy de Lôme (IRDL), UMR CNRS 6027, ENSTA Bretagne, France

B. Huneau

Institut de Recherche en Génie Civil et Mécanique (GeM), UMR CNRS 6183, Ecole Centrale de Nantes, France

C. Champy \& P. Charrier

Vibracoustic, CAE \& Durability Prediction, Carquefou, France

\begin{abstract}
The fatigue properties of filled elastomers are strongly related to the inclusions' population induced by their complex recipes and the successive stages of the manufacturing process (mixing, injection and curing). The improvement of these properties involves at first an ability to describe the statistical features of these inclusions' population in terms of size, geometry, nature and spatial distribution. Then a detailed understanding of the damage mechanisms is required in order to define the mechanical criticality of inclusions according to their characteristics under cyclic loading. The aim of the study is to take advantage of tomography observations that enable a three dimensional vision to access the statistical description of the inclusions population and of the related damage mechanisms. First of all, a tool to detect and characterize accurately inclusions and cracks is presented. Then, this tool is applied to an interrupted specimen in fatigue, for a compound filled with carbon black. A statistical analysis is performed on the nature of inclusions leading to cracks, on the kind of damage mechanisms (polar crack, cavitation between 2 inclusions or failure of an inclusion) and on the density and the geometric properties of activated inclusions. An additional focus is given on the inclusions leading to the biggest cracks in order to assess more precisely the features (nature, size......) of the most critical inclusions for fatigue.
\end{abstract}

\section{INTRODUCTION}

The industrial development of elastomers resistant to fatigue is very complex because the parameters driving the microstructure are incredibly numerous, including the original ingredients and the processing parameters, from mixing to injection and curing.

In elastomeric parts, the fatigue cracks are initiated either at the parting line, which can be considered as a geometrical defect acting as a strain concentrator, or around inclusions. In carbon black filled natural rubber, the initiation around inclusions seems to prevail (Masquelier 2014). In the literature, different inclusionary initiation mechanisms are distinguished (Gent et al. 1984, Le Saux et al. 2011, Le Gorgu Jago 2012, Le Cam et al. 2013): inclusions failure, cavitation at one pole or between close inclusions and debonding at one or both poles. The mechanism is strongly related to the microstructure features such as the inclusions modulus, their shape, size and spatial distribution and the cohesion and adhesion properties. Huneau et al. (2016) described the inclusions which induced micro-cracks during fatigue loading thanks to observations of interrupted fatigued samples and fatigue fracture surfaces using a scanning electron microscope. The study highlights that fatigue damage generally initiates on carbon black agglomerates or on metallic oxides such as $\mathrm{ZnO}$. It was shown that the ones on carbon black agglomerates are almost always followed by a crack propagation leading to the failure of the sample. Scanning Electron Microscopy (SEM) observations enable the analysis of debonding and failure mechanisms but not cavitation mechanism. Moreover, the generation of statistical results requires a huge number of observations. Thus, this experimental protocol is time consuming and difficult to apply on a lot of compounds. Another way to assess the fatigue damage evolution can be X-ray micro-tomography (Le Gorgu Jago 2008, Le Gorgu Jago 2012, Le Saux et al. 2011). In this case, a lot of inclusions and cracks can be observed within the analysis of one sample and for a rather large volume compared to SEM. The treatment used is almost always a thresholding method of the gray levels in order to have the size and the number of cracks or of the 
inclusions, usually denser than the matrix. Then, the initiation mechanisms and the damage evolution are identified from the geometry of the detected cracks and inclusions.

The aim of this study is to provide a well resolved and statistical description of inclusions and the related micro-cracks. First of all, the experimental set-up to characterize the initiation mechanisms is presented. Then, a tool is developed to provide an accurate description of the microstructure features of both inclusions and cracks. Finally, the results obtained for a natural rubber filled with carbon black are presented and the conclusions on the inclusions criticality are highlighted.

\section{EXPERIMENTAL SETTINGS}

\subsection{Materials and experimental protocol}

The material studied is a fully formulated natural rubber reinforced with carbon black. Classic hourglass samples are used to characterize the fatigue properties. These samples are injected and representative of the manufacturing process used for automotive anti-vibration parts.

The damage evolution is followed thanks to the observations of interrupted fatigued samples, as presented in Huneau et al. (2016). First of all, the Wöhler curve for relaxing testing conditions is determined for the compound. The number of cycles at initiation (Ni) is defined according to the loss of specimen stiffness (Ostoja-Koczynski 2003). Then, for the interrupted tests, the nominal maximal principal strain is set to $200 \%$ and the imposed amount of cycles is chosen as $60 \%$ of the number of cycles leading to initiation (Ni). (Fig. 1).

\subsection{Experimental devices}

Micro-tomography is a remarkable tool to provide a non-destructive internal observation. This method

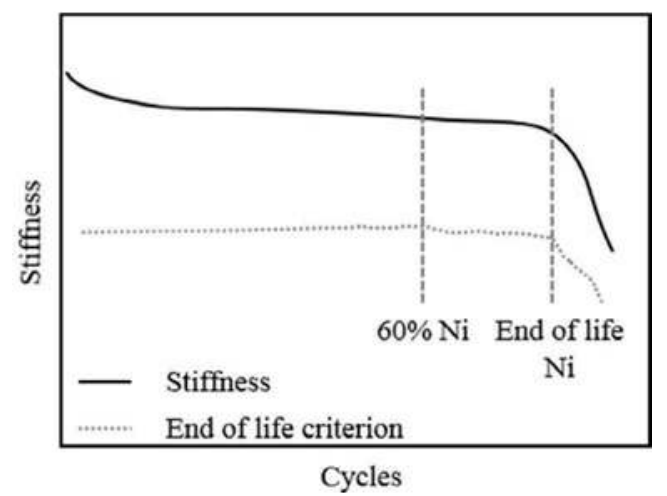

Figure 1. Evolution of the stiffness versus the number of cycles (X-log scale).

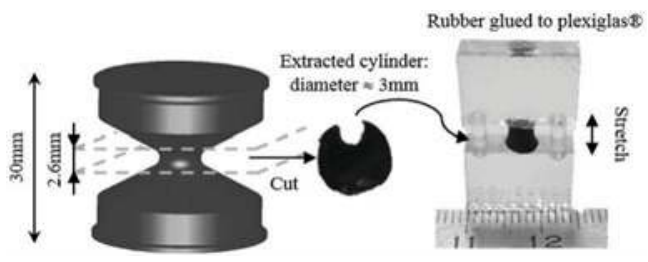

Figure 2. Specimen extracted from the hourglass sample and stretched.

has been widely used for the study of materials microstructure (Salvo 2003) including rubber. Compared with usual 2D analysis methods such as the SEM, the 3D reconstruction of the microstructure by micro-tomography grants to:

- Quantify statistically the number, size, shape and orientation of objects;

- Study the spatial distribution of objects;

- Avoid artefacts due to cutting and surface preparation for 2D observations.

However, the micro-tomography has some drawbacks:

- Resolution is about a few micrometers (much poorer than the resolution of SEM);

- Acquisition can demand long durations;

- Result files can be of huge size;

- Lack of access to the chemical composition (unlike SEM with EDS).

Furthermore, the X-ray micro-tomography is an excellent tool to follow in a 3D way the damage evolution (Le Gorgu Jago 2012, Le Saux et al. 2011). The sample of the study was analyzed with an XRadia MicroXCT-400 micro-tomograph, located at Ecole Centrale de Nantes (GeM). In order to have a high enough resolution, the volume of the analyzed hourglass sample was reduced.

\subsection{Samples}

The sample observed by micro-tomography is a small cylinder (diameter $\approx 3 \mathrm{~mm}$ ) extracted at the middle height of the interrupted fatigue sample. A resolution of $1.7 \mu \mathrm{m}$ is reached with this small sample. In order to open the cracks, the sample's faces were glued to plexiglas $®$ plates and the sample was stretched thanks to small plexiglas ${ }^{\circledR}$ pins (Fig. 2).

\section{ANALYSIS PROTOCOL}

\subsection{Images processing}

Many softwares are available to process and visualize 3D micro-tomography images, such as Avizo3D, myVGL, Paraview and ImageJ. However, our choice was to select the Python programming language 
which is widely used in the scientific world and can provide access to more specific developments. Indeed, the use of a programming language for tomography processing allows finer control and more complex analysis possibilities. Gouillart et al. (2016) show the ability of Python and the scikit-image library to analyse micro-tomography data.

The goal of the images processing is the segmentation of the different types of objects (inclusions and voids) in the material. The segmentation consists in partitioning the tomography into several regions, in which the voxels satisfy a given homogeneity criterion, based for example on the gray level. The segmentation algorithm must minimize the loss of information on the microstructure caused by the simplification of the images, due to the different applied transformations. Thus, segmentation remains a difficult step since the choice of the segmentation algorithm can lead to significant differences in the morphological characterization of the microstructure. The used algorithms to segment regions of interest depend on the properties of the images. They can be classified in different families such as the thresholding algorithms (Otsu 1979), the region growing algorithms (Grady 2016) and the active contour algorithms (Kass 1988). Thresholding methods are the most common. They use the histogram data of the images. The threshold gray level is chosen manually or automatically to select the objects of interest. These techniques consider voxels independently and do not use their spatial information. These techniques are effective when the objects and the background have two distinct peaks on the grayscale histogram. This segmentation approach was used by Le Gorgu Jago (2012) and Le Saux et al. (2011) to detect inclusions denser than the matrix and cavities inside the specimens, in order to assess the damage evolution. In this paper another segmentation method is used. First of all, a pre-treatment is applied to the reconstructed data, then, the inclusions and cracks are detected thanks to a region growing algorithm.

\subsubsection{Pre-treatment}

An exposure adjustment is performed in order to increase the contrast and delete extreme values due to the experimental noise. For practical reasons, the gray levels are set between 0 and 1 ( 0 : white voxels and 1: black voxels). Then, a 3D median filter is applied to make the segmentation easier afterwards. It reduces noise while preserving edges. The mask of the filter is chosen small $(3 \times 3 \times 3$ voxels $)$ in order to avoid deleting small inclusions.

\subsubsection{Inclusions segmentation}

The classical thresholding method is not used here for the inclusions segmentation because the choice of the threshold value (i.e. the gray value) is sensitive. Indeed, the chosen threshold has a strong influence on the shape and size of the biggest detected inclusions (Fig. 3).

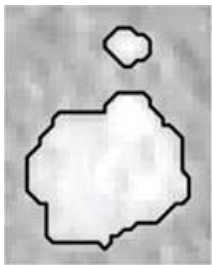

Threshold $=0.85$ $\mathrm{d}_{\mathrm{eq}}=246 \mu \mathrm{m}$

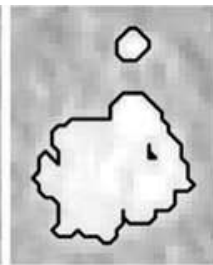

Threshold $=0.9$ $\mathrm{d}_{\mathrm{eq}}=223 \mu \mathrm{m}$

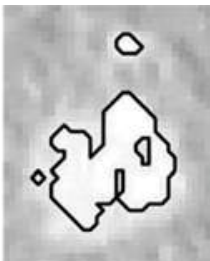

Threshold $=0.95$ $\mathrm{d}_{\mathrm{eq}}=183 \mu \mathrm{m}$
Figure 3. Effect of the threshold value for thresholding algorithm on the inclusion shape and size $\left(\mathrm{d}_{\mathrm{eq}}\right.$ : equivalent diameter of the sphere corresponding to the same volume).

Moreover, the bright inclusions do not create a specific peak on the grayscale histogram (Fig. 4). Thus, in order to have an accurate inclusions segmentation, a 3D region growth algorithm ("random walk") that combines information on the value of the gray level and the position of the voxels is used (Grady 2016). First of all, the user defines markers labeling regions of the image belonging clearly either to the inclusion or to the matrix. Some regions remain indeterminate like highlighted in the Figure 4. Then, an anisotropic diffusion equation is solved with tracers initiated at the marker's positions. The local diffusivity coefficient is defined as higher if neighboring voxels have similar values, so that diffusion is difficult across high gradients. The label of each unknown voxel is set to the label of the known marker that has the highest probability to be reached first during the diffusion process.

The matrix value is set as the one for which the curve of the grayscale histogram of the whole tomography deviates from the line drawn (i.e. 0.77) (see the top right scheme in Fig. 4). It corresponds to the
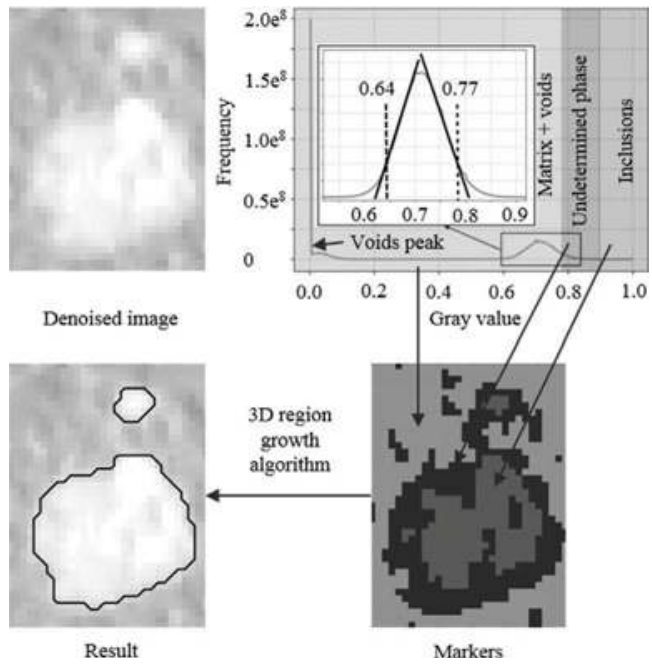

Figure 4. Inclusions segmentation steps and result of the algorithm superposed to the denoised data. 


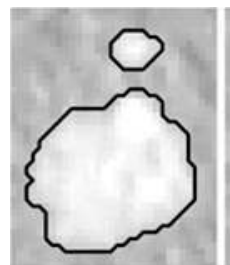

Thr. inclusions $=0.85$ $\mathrm{d}_{\mathrm{eq}}=259 \mu \mathrm{m}$

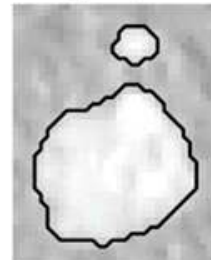

Thr. matrix $=0.75$

$\mathrm{d}_{\mathrm{eq}}=252 \mu \mathrm{m}$

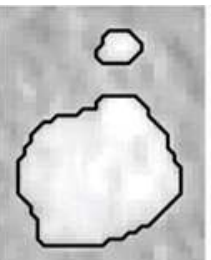

Thr, inclusions $=0.9$ $\mathrm{d}_{\mathrm{eq}}=248 \mu \mathrm{m}$

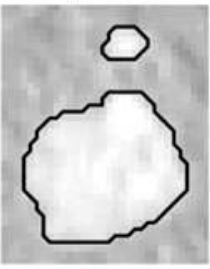

Thr. matrix $=0.77$ $\mathrm{d}_{\mathrm{eq}}=248 \mu \mathrm{m}$
Thr. inclusions $=0.95$ $\mathrm{d}_{\mathrm{eq}}=235 \mu \mathrm{m}$

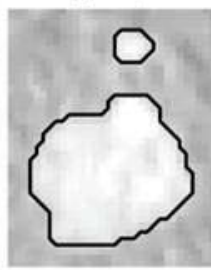

Thr. matrix $=0.79$ $\mathrm{d}_{\mathrm{eq}}=245 \mu \mathrm{m}$

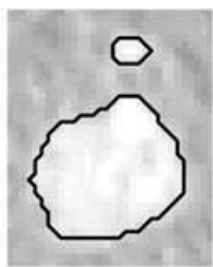

Figure 5. Effect of the inclusions and matrix threshold values for regions growth algorithm on the inclusions shape and size.

end of the matrix peak. The value of inclusions is chosen arbitrary as equal to 0.9 .

Thanks to this segmentation method, the size of the biggest inclusions is almost insensitive to the markers defined by the user. Indeed, the Figure 5 shows that the threshold value of inclusions has little influence on the shape and size of the biggest inclusions, unlike conventional thresholding algorithms. Moreover, the choice of the matrix markers has almost no effects on the results (Fig. 5).

Finally, only inclusions with more than 27 voxels (i.e. size of the filter $3 \times 3 \times 3$ voxels) are kept. Consequently, the smallest inclusions have an equivalent diameter of $6.3 \mu \mathrm{m}$.

It should also be underlined that the contrast observed thanks to micro-tomography is highly dependent on the chemical nature of the inclusions. In this study, the metallic oxide inclusions are identified clearly but it was not possible to do so with the carbon black inclusions (except for the biggest ones).

\subsubsection{Sample and cracks segmentation}

In that particular case, a conventional thresholding operation is applied in order to separate it from the background because the voids and the matrix have two distinct peaks on the grayscale histogram (see top right figure in Fig. 4). The threshold value is set at middle distance between those two peaks (i.e. $0.36)$. The cracks are detected with the same regions growth algorithm than inclusions. The voids markers are set to the middle distance between the matrix and voids peaks (i.e. 0.36). The matrix markers for the crack detection are set like for inclusions but this time at the beginning of the matrix peak (i.e. 0.64) (Fig. 4). The outer cracks are not considered in this

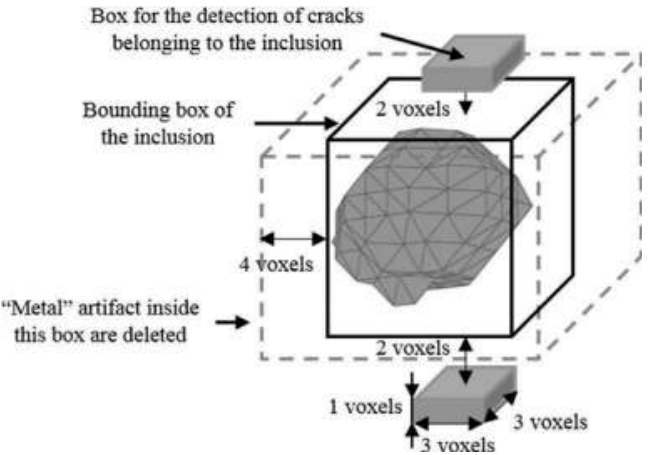

Figure 6. Strategy of association of cracks with inclusions and removal of "metal artifact".

study because the micro-tomography images have a thin bright line around the border of the specimen. Thus, in order to delete this contour, the specimen is eroded of a few voxels. Only inclusions and cracks inside this eroded volume are kept.

\subsection{Combination of inclusions and cracks}

Once the inclusions and cracks are detected, the next step is to associate them. A specific algorithm has also been developed (Fig. 6) to detect the cracks in the vicinity of one inclusion's pole. Special attention to artifacts induced by metallic oxides, such as $\mathrm{ZnO}$ (Le Gorgu Jago 2012) has to be paid, in order to not identify them as voids. This "metal artifact" appears only in $(\mathrm{x}, \mathrm{y})$ plane of the tomography and close to metallic oxides. Thus, the identified voxels as voids but located in the immediate vicinity of the inclusions are not considered (Fig. 6).

At the end of this process, it is possible to identify if a crack is located at one of an inclusion's poles or if the cracks are detected without inclusions nearby, and, reversely, if some inclusions present no cracks.

\subsection{Combination with the mechanical states in the sample}

The complex geometry of the AE2 sample leads to different local strains and stresses. Relating the damage description to the mechanical state therefore requires a specific analysis. Figure 7 shows the identified strain field in the sample thanks to a FE numerical simulation. It can be observed that, in the volume of the cylinder cut for the micro-tomography observations, the strain is almost constant along the sample axis ( $\mathrm{Z}$ axis on Figure 7 ). It was therefore decided to analyze the sample according to radial slices (see Figure 7).

To do so, the outline of the specimen is detected for each slice of the tomography (in the planes XY), and then polygonised. Once the exterior edge of the sample detected, 6 volumes are defined and the corresponding strains states coming from the FE simulations associated. 


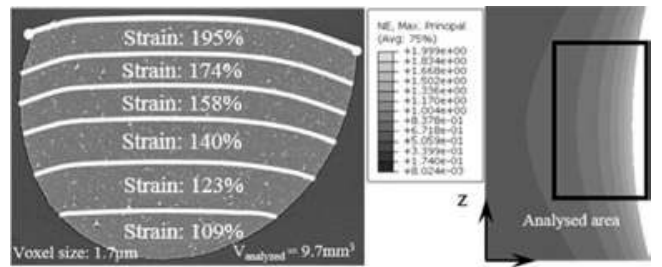

Figure 7. Mechanical fields in the sample analyzed.

\subsection{Computation}

As evoked in the introduction, the manipulation and the analysis of tomographic data raise several challenges related to the calculation time and the management of the memory for the images processing. In our case, the python script is run on a powerful cluster and the growing regions algorithm is computed using multiprocessing. To do so, the volume is split into 54 subvolumes with a covering rate of $50 \%$.

The volume of investigated material is around $9.7 \mathrm{~mm}^{3}$. Then, each detected inclusion can be morphologically (size, shape, spatial distribution and orientation) analyzed (Glanowski et al. 2018). To give an idea of the statistical relevancy of the analysis, the images processing gave access to the features of 118640 metallic oxides.

\section{RESULTS}

\subsection{Nature of inclusions leading to cracks}

The first question arising concerns the chemical natures of the inclusions leading to cracks. As explained previously, only metallic oxides inclusions were detected directly here. As cracks are initiated on inclusions, the assumption is made here that cracks detected without any associated inclusions are due to carbon black agglomerates. The associated cracks without inclusions or with a metallic oxide are counted for each defined strain zone. As regions have

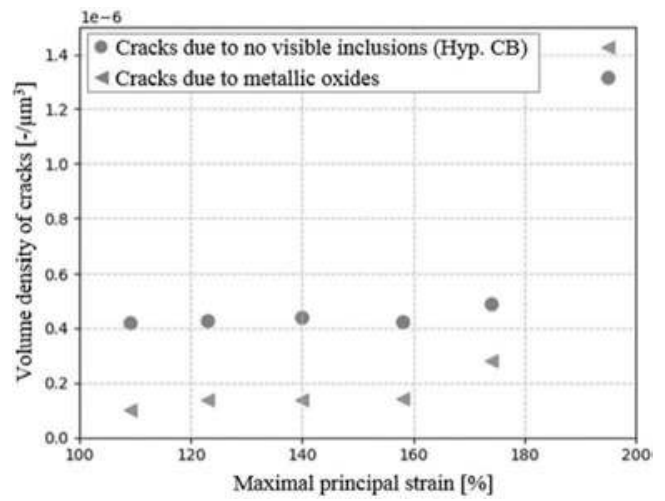

Figure 8. Volume density of cracks for the different zones.

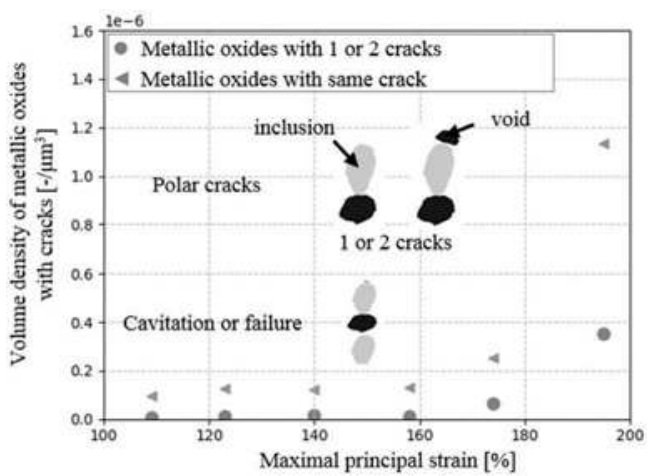

Figure 9. Volume density of metallic oxides with cracks with respect to the maximal principal strain for the various fatigue crack initiation mechanisms.

not the same volume, the volume density of cracks, defined as the number of cracks divided by the analyzed volume, is followed rather than the number of cracks. Figure 8 highlights that both the cracks volume densities without inclusions (possibly due to carbon black agglomerates) and associated to metallic oxides, increase with strain level. A sudden increase is visible for a nominal principal strain of $200 \%$.

\subsection{Mechanisms}

The understanding of the initiation mechanisms is important for the industrial development of the materials, in order to find a way to delay them. The analysis tool developed here gives access to polar cracks (that could be induced by interface debonding or cavitation near the poles) and cracks due to several inclusions (induced either by the cavitation between close inclusions or by the failure of inclusions (Le Saux et al. 2011)). Figure 9 presents the evolutions of these two populations of inclusions. First, it can be concluded that more than $75 \%$ of the cracks are polar ones. Another interesting observation is that the cracks

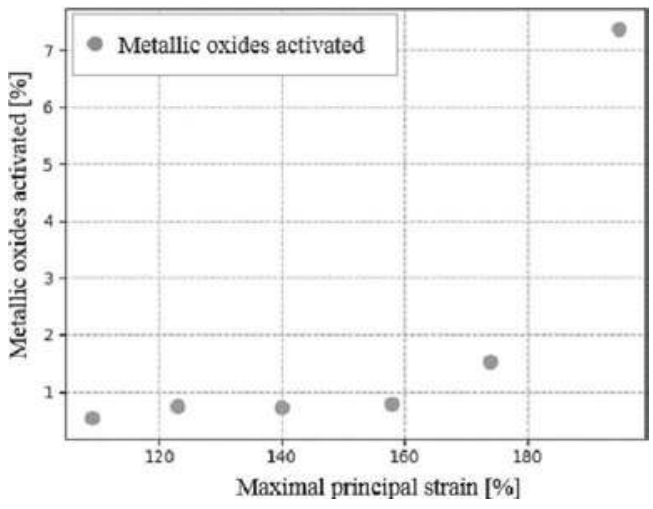

Figure 10. Mechanical fields in the specimen. 


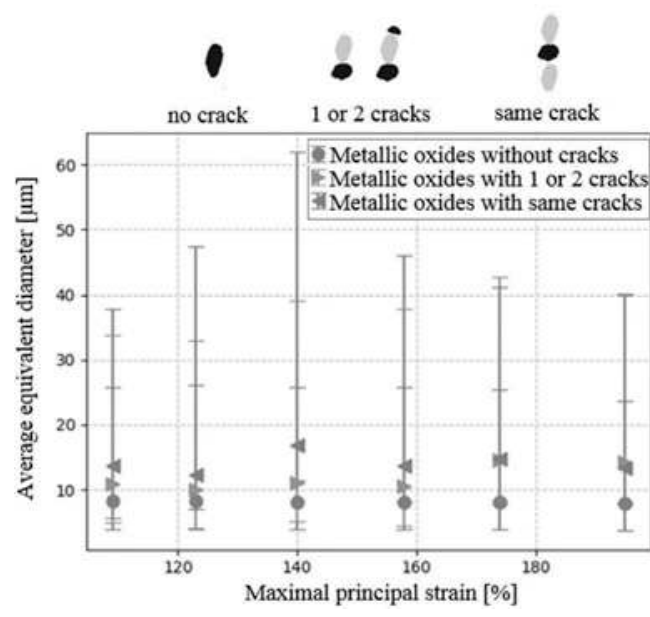

Figure 11. Size of metallic oxides with and without cracks.

involving two inclusions are detected only for the highest nominal strains.

\subsection{Volume density of activated metallic oxides}

The analysis over a large number of inclusions also offers access to the percentage of metallic oxides that are activated (i.e. inducing one or two cracks). Figure 10 shows that the percentage of metallic oxides activated increases with strain.

\subsection{Average size of metallic oxides with and without cracks}

Finally, one of the most import information for the industrial development of these materials is the size of metallic oxides that would not induce any cracks during considered fatigue loads. Figure 11 shows the equivalent diameter of metallic oxides for 3 cases: presenting no cracks, with polar cracks and with cracks related to two inclusions. For each case, the median value is given and the interval is set between the $5^{\text {th }}$ and the $95^{\text {th }}$ percentiles. The metallic oxides presenting no cracks have a median value lower than the ones with cracks in any configuration. Moreover, these metallic oxides exhibit a median value independent on the strain level. It means that metallic oxides with an equivalent diameter below $9 \mu \mathrm{m}$ do not create micro-cracks in the present case. Another observation is that the inclusions related to a same crack exhibit a higher median value than those with polar cracks, except for the highest strain levels.

\section{CONCLUSIONS}

This paper aimed at providing quantitative data on the relation between inclusions and the early stages of micro-cracks initiation. X-ray micro-tomography data have been used and specific images processing tools have been developed. The region growth algorithm of Grady (2016) is used in this study in order to detect accurately inclusions and voids. This kind of algorithm has the main advantage to be less dependent on parameters value, compared to thresholding approach. The analysis provided statistical 3D data at a scale that seems relevant (below $10 \mu \mathrm{m}$ for cracks and inclusions). The geometric features (size, orientation and shape) of the inclusions in the material leading or not to one or several cracks are indeed described. It was found that metallic oxides with an equivalent diameter below $9 \mu \mathrm{m}$ do not create micro-cracks. Thus, this size could be set as an objective for the mixing stage in order to improve the compounds duration life. Another observation is that more than $75 \%$ of the cracks initiated on metallic oxides are polar cracks associated with only one inclusion. It was also observed that the cracks generated by metallic oxides (over the minimum detected size) represent around $25 \%$ of the total number of detected cracks, except for the highest strains, for which is a $50-50 \%$ share. The generated data are clearly appealing and calls for further analysis.

\section{ACKNOWLEDGEMENTS}

The authors would like to thank the ANRT for its financial support and François Bertrand (GeM) for performing the micro-tomography measurements.

\section{REFERENCES}

Gent A. N. and Park B., 1984, Journal of Materials Science, 19, 1947-1956.

Glanowski T., Huneau B., Marco Y., Le Saux V., Champy C., Charrier P., 2018, Fatigue 2018, 165.

Gouillart E., Nunez-Iglesias J. and van der Walt S., 2016, Adv. Struct. Chem. Imag, 2:18.

Grady L., 2016, IEEE trans. Pattern Analysis and Machine Intelligence, 28, 1768-1783.

Huneau B., Masquelier I., Marco Y., Le Saux V., Noizet S., Schiel C. and Charrier P., 2016, Rubber Chemistry and Technology, 89, 126-141.

Kass M., Witkin A., Terzopoulos D., 1988, International Journal of Computer Vision, 1, 321-331.

Le Cam J.-B., Huneau B. and Verron E., 2013, International Journal of Fatigue, 52, 82-94.

Le Gorgu Jago K., 2008, ECCMR V, 173-177.

Le Gorgu Jago K., 2012, Rubber Chemistry and Technology, 85, 387-407.

Le Saux V., Marco Y., Calloch S. and Charrier P., 2011, Polymer Eng. and Science, 51, 1253-1263.

Masquelier I., 2014, PhD thesis, Université de Bretagne Occidentale.

Otsu N., 1979, IEEE Transactions on Systems, Man and Cybernetics, 9, 62-66.

Kuczynski O., Charrier P. et al. 2003, ECCMR.

Pyrz R., 2004, Mechanics of Microstructural Materials, 173-233.

Salvo L., Cloetens P., Maire E., 2003, Nucl. Instrum. Meth., 200B, 273-286. 